\title{
Evaluación del serodiagnóstico en el absceso hepático amebiano
}

\author{
Luis C. Orozco ${ }^{1}$, Claudia M. Trujillo ${ }^{2}$, Herman J. Arteaga ${ }^{3}$, Luis A. Villar ${ }^{3}$, Luis F. Burbano ${ }^{3}$, \\ Paola J. Contreras ${ }^{3}$, Claudia V. Leal ${ }^{3}$
}

\begin{abstract}
Resumen
El presente estudio fue diseñado para evaluar la eficacia del diagnóstico serológico del absceso hepático amibiano (AHA). Se utilizó una prueba de ELISA para la detección en suero de IgG específica, utilizando una fase sólida con una alta capacidad de adherencia.
\end{abstract}

Se estudiaron 147 personas; 22 pacientes con sospecha clínica y ultrasonográfica de $\mathrm{AHA}, 30$ individuos completamente sanos, 9 portadores asintomáticos de E. histolytica, 35 con colitis amebiana pasada, 35 con otras parasitosis intestinales, 9 con otras patologías hepáticas y 6 con colitis amebiana presente. El rendimiento global de la prueba fue analizado por medio de las curvas del receptor-operador y del área bajo la curva. Se determinó que existe una diferencia significativa en la densidad óptica (DO) de los siete grupos estudiados (Kruskal-Wallis entre todos los grupos: $p=0,0001$ ); esta diferencia no existe cuando, al comparar, se elimina el grupo 1 del análisis (Kruskal-Wallis exceptuando el grupo 1: $p=0,8203$ ). El área bajo la curva ROC fue igual a 0,9941, dato muy cercano al ideal que es 1 .

Se recomienda la utilización de esta fase sólida teniendo en cuenta la importancia de la fase de bloqueo con albúmina de huevo. Se determinó que, a pesar de estar en una zona donde el diagnóstico de amebiasis es frecuente, las infecciones intestinales por esta ameba no presentan niveles detectables de lgG específica contra $E$. histolytica por esta prueba. Sin embargo, es importante ampliar la cantidad de personas estudiadas en estos grupos, principalmente, en el grupo de pacientes con otras patologías hepáticas puesto que este grupo incluye las entidades clínicas con las que realmente se debe hacer el diagnóstico diferencial de (AHA) (absceso hepático piógeno (AHP), principalmente) para poder realizar la última fase de la evaluación. El estudio de este grupo nos ayudaría a obtener valores reales de sensibilidad, especificidad y valores predictivos y nos darían una estimación real de la capacidad discriminatoria de la prueba para obtener el verdadero valor del área bajo la curva y no el casi ideal que informamos aquí.

\section{Summary}

This study was carried out to evaluate the diagnostic efficacy of a serological test for amebic liver abscess (ALA) in an endemic amoebiasis area. An enzyme-linked immunosorbent assay (ELISA) to detect specific IgG against Entamoeba histolytica using a solid phase with high adherent capacity was applied.

\footnotetext{
Escuela de Enfermería, Universidad Industrial de Santander, Bucaramanga, Colombia.

CINTROP, Universidad Industrial de Santander, Bucaramanga, Colombia.

Escuela de Medicina, Universidad Industrial de Santander, Bucaramanga, Colombia.
} 
Serum samples were obtained from 147 individuals: 22 patients with amoebic liver abscess diagnosed on the basis of clinical symptoms and ultrasound examination, 30 healthy individuals, 9 asymptomatic $E$. histolytica infections, 35 with past episodes of amebic colitis, 35 with other parasitic infections, 9 with other hepatic ailments and 6 with amebic dysentery. Test performance evaluation was analyzed by the relative operating characteristic $(\mathrm{ROC})$ curves and area under the curve. We found a difference between the optical density values of the groups (Kruskal-Wallis between all the groups: $p=0.0001$ ), this difference disappeared when the 1 was excluded from the analysis (Kruskal-Wallis without group 1: $\mathrm{p}=0.8203$ ). Area under ROC curve was 0.9941 .

We suggest the use of this solid phase, taking care to blocki the plates with egg albumin. Although the study was made in an area where amebiasis diagnosis is common, individuals with intestinal asymptomatic amebiasis did not present levels of specific lgG by the evaluated test. However, to carry out the last evaluation phase, it is important to increase the number of samples in all the groups especially in the group with other hepatic ailments because these are the clinical states from which ALA has to be differentiated when the test is applied in clinical use. This phase can provide a real estimate of test's sensitivity, specificity and predictive values and a more realistic area under the ROC curve.

La amebiasis invasora sintomática se desarrolla en $10 \%$ de los individuos infectados por Entamoeba histolytica, causando más de 100.000 muertes anuales (1). El absceso hepático amebiano (AHA) es una de las presentaciones más graves de la amebiasis invasora. Como causa de admisión hospitalaria en zonas endémicas, el AHA contribuye con 0,1 a $1,2 \%$ de los pacientes (2).

El diagnóstico definitivo de dicha entidad se realiza por el hallazgo de los trofozoítos característicos de E. histolytica en el material obtenido del absceso ya sea por biopsia o por aspiración hepática. Los métodos de diagnóstico parasitológico más utilizados son el examen directo, los cultivos y las tinciones. Estos métodos son de una tecnología sencilla, pero, además de necesitar de un método invasor para la obtención de la muestra, son muy subjetivos y de baja sensibili$\operatorname{dad}(3,4)$.

Por esta razón, los métodos serológicos han cobrado importancia ya que, además de ser fáciles de realizar, rápidos y económicos, permiten, al igual que los métodos parasitológicos, el diagnóstico diferencial de AHA con otras entidades que pueden dar imágenes ultrasonográficas y síntomas semejantes como el absceso hepático piógeno (AHP) (3-10).
El método más utilizado actualmente para el serodiagnóstico de AHA es el ensayo inmunoenzimático (ELISA) debido a su alta reproducibilidad, objetividad en la lectura de los resultados y a la facilidad para procesar gran cantidad de muestras al mismo tiempo. Por este método, la detección de IgM específica contra $E$. histolytica utilizando extracto antigénico total, permite establecer el diagnóstico con una especificidad aproximada de $100 \%$, pero, su sensibilidad no supera $80 \%$ probablemente debido a las dificultades técnicas que presenta su determinación $(4-6,11)$. Por otra parte, la detección de IgG específica por la misma técnica, a pesar de su gran sensibilidad, presenta algunos inconvenientes particularmente en zonas endémicas para amebiasis. En estas áreas, pueden presentar niveles detectables de IgG específica individuos que hayan sufrido de colitis amebiana o individuos sanos portadores de quistes (3-6, 8-12).

En Colombia, la segunda encuesta nacional de morbilidad reveló una prevalencia de infección por E. histolytica de $12,1 \%$ (2). No obstante, se estima que este dato puede llegar a $50 \%$ en algunas áreas donde las condiciones de higiene y saneamiento ambiental son deficientes (1).

El presente estudio fue diseñado para evaluar la eficacia del diagnóstico serológico del AHA en 
una región donde la infección por E. histolytica es frecuente. Se utilizó una prueba de ELISA para la detección de IgG específica en suero, utilizando una fase sólida con una alta capacidad de adherencia.

\section{Materiales y métodos \\ Diseño}

El diseño corresponde a una fase III-IV según la clasificación de Nierenberg y Feinstein (13).

\section{Grupos de estudio}

Se estudiaron 146 personas. El suero obtenido de cada una de ellas fue dividido en alícuotas y almacenado a $-20^{\circ} \mathrm{C}$ hasta su utilización. Adicionalmente, a los controles sanos se les aplicó previamente una encuesta para analizar los antecedentes de contacto con E. histolytica (episodios pasados de disentería o colitis amebiana crónica) y se les realizó un coprológico seriado con el método de concentración de formol-éter.

Los sueros analizados provenían de los siguientes grupos de personas:

Grupo 1: 22 pacientes atendidos en el Hospital Universitario Ramón Gonzalez Valencia (HURGV) y en el Instituto Nacional de Salud, con sospecha clínica y ultrasonográfica de AHA.

Grupo 2: 30 individuos sanos, sin antecedentes de colitis amebiana, cuyo estudio coprológico resultó negativo para parásitos intestinales.

Grupo 3: 9 individuos sanos, sin antecedentes de colitis amebiana, pero con presencia de quistes de E. histolytica en el estudio coprológico.

Grupo 4: 35 individuos sanos, con antecedentes de colitis amebiana, cuyo estudio coprológico resultó negativo para otros parásitos intestinales patógenos aparte de E. histolytica.

Grupo 5: 35 individuos sanos, sin antecedentes de colitis amebiana, pero con presencia de otros parásitos intestinales en el estudio coprológico (Blastocystis hominis, Endolimax nana, Entamoeba hartmanni, Entamoeba coli, Chilomastix mesnili, lodamoeba butschlii, Ascaris lumbricoides, Trichuris trichiura y uncinaria).

Grupo 6: 9 pacientes del HURGV con patología hepática diferente al AHA (abscesos hepáticos piógenos, 4; cáncer de hígado, 1 ; cirrosis hepática, 1 ; ictericia obstructiva por colelitiasis, 3 ).

Grupo 7: 6 individuos con colitis amebiana diagnosticada clínica y parasitológicamente (presencia de trofozoítos hematófagos de E. histolytica en las heces).

\section{ELISA para detección de IgG específica contra E. histolytica en suero}

Antígeno: el antígeno fue preparado por Behring Institut (Behringwerke AG, Marburg, Alemania) utilizando cultivos axénicos de trofozoítos de $E$. histolytica, cepa HK-9, con una concentración final de proteínas de $1 \mathrm{mg} / \mathrm{mL}$.

Procedimiento: siguiendo la técnica general descrita por Engvall y Perlmann (1972):

1. Se utilizaron platos Immulon 4 (Dynatech Laboratories Inc.) como fase sólida (los platos Immulon 4 son fabricados en poliestireno modificado con un tratamiento especial para realzar su unión a proteínas).

Estos platos fueron sensibilizados con $100 \mathrm{uL} /$ pozo de antígeno diluido en solución tamponada de carbonatos $50 \mathrm{mM}, \mathrm{pH} 9,5$, a una concentración de trabajo de $3 \mathrm{ug} / \mathrm{mL}$, incubando 2 horas a $37^{\circ} \mathrm{C}$ en cámara húmeda.

2. Se descartó el liquido de los pozos y se realizaron cinco lavados con solución tamponada de fosfatos salinos $30 \mathrm{mM}, \mathrm{pH} 7,2$, que contenía 0,05\% de Tween 20 (PBST).

3. El bloqueo de los sitios libres de antígeno en la fase sólida se realizó con PBST con albúmina de huevo (egg whites, Sigma Chemical Co.) al $5 \%$ incubando $2 \mathrm{~h}$ a $37^{\circ} \mathrm{C}$ en camara húmeda.

\section{Se lavó nuevamente como en el paso 2.}

5. Las diluciones de los sueros se realizaron en PBST albúmina de huevo al 3\% (PBSTA) incubando $100 \mathrm{uL} /$ pozo de la dilución, $1 \mathrm{~h}$ a $37^{\circ} \mathrm{C}$ en camara húmeda a la oscuridad.

\section{Lavado.}

7. La dilución de conjugado (anticuerpos de cabra anti-IgG humana marcados con fosfatasa alcalina, Sigma Chemical Co.) se realizó en las mismas condiciones de la dilución de suero. 
8. Lavado.

9. Se agregaron $100 \mathrm{uL} /$ pozo del sustrato de la enzima (o-nitrofenilfosfato, Sigma Chemical Co.) diluido a $1 \mathrm{mg} / \mathrm{mL}$ en solución tamponada de dietanolamina $0,1 \mathrm{M}, \mathrm{pH} 9,8$, incubando $25 \mathrm{~min}$ a $27^{\circ} \mathrm{C}$.

\section{Se agregaron $50 \mathrm{uL} /$ pozo de $\mathrm{NaOH} 3 \mathrm{M}$.}

11. Las densidades ópticas (DO) se determinaron a una longitud de onda de $405 \mathrm{~nm}$ en un lector para ELISA, EL301 Bio-tek Instruments Inc. Todas las muestras se procesaron por duplicado y se utilizó el promedio como dato.

\section{Titulación de suero y conjugado enzimático}

Usando la concentración de antígeno de $3 \mathrm{ug} /$ $\mathrm{mL}$, se realizaron diluciones de suero de 1:500 a 1:8.000 y diluciones de conjugado de 1:500 a 1:4.000. La óptima dilución de suero y conjugado enzimático se escogió teniendo en cuenta los siguientes criterios:

1. Nivel de discriminación positivo-negativo: dilución a la cual se obtenían las mayores diferencias entre las DO del pool de sueros positivos (grupo 1) y el pool de sueros negativos (grupo 2).

2. DO del pool de sueros positivo: dilución que daba lecturas aproximadas a 1,5 DO (lector con densidades ópticas entre 0 y 3 ).

DO de fondo: dilución que registraba las menores lecturas de DO en los blancos utilizados para evaluar las uniones inespecíficas del pool de sueros positivo y negativo o del conjugado a la fase sólida después del bloqueo o uniones inespecíficas del conjugado a componentes antigénicos.

\section{Métodos estadísticos de análisis de datos}

Para comparar las densidades ópticas de los diferentes grupos, se utilizó la prueba de KruskalWallis. Para evaluar si existe una distribución bimodal, se realizó una gráfica de distribución de frecuencias. El rendimiento global de la prueba fue analizado por medio de las curvas del receptor-operador y el área bajo la curva $(14,15)$; se realizaron dos curvas, una con los valores obtenidos y otra con los valores agrupados por 0,05 de intervalo.

\section{Resultados}

\section{Optima dilución de suero y conjugado enzimático}

Se estableció 1:1.000 como la óptima dilución de suero y conjugado enzimático de la prueba teniendo en cuenta que al utilizar estas diluciones se obtuvieron lecturas de densidad óptica cercanas a $1,5(1,383 \mathrm{DO})$ y el nivel de discriminación positivo-negativo fue de 1,186 DO sin aumento considerable en la densidad óptica de fondo.

\section{Distribución de las densidades ópticas en los grupos de pacientes}

La distribución de los datos, los valores promedio de DO y las desviaciones estándar de los grupos estudiados se muestran en el cuadro 1. Existe una diferencia significativa en las DO de los 7 grupos estudiados (Kruskal-Wallis entre todos los grupos: $p=0,0001$ ); esta diferencia no existe cuando, al comparar, se elimina el grupo 1 del análisis (Kruskal-Wallis, exceptuando el grupo 1: $\mathrm{p}=0,8203$ ).

Cuadro 1. Densidades ópticas de los diferentes grupos de pacientes.

\begin{tabular}{|c|c|c|c|c|c|c|c|c|}
\hline Grupo & $\mathrm{n}$ & Min & Max & Q1 & $\mathrm{Q} 2$ & Q3 & $\bar{X}$ & $\mathrm{DE}$ \\
\hline 1 & 22 & 0,199 & 1,835 & 1,123 & 1,358 & 1,442 & 1,230 & 0,376 \\
\hline 2 & 30 & 0,136 & 0,358 & 0,152 & 0,160 & 0,181 & 0,174 & 0,043 \\
\hline 3 & 9 & 0,144 & 0,271 & 0,155 & 0,162 & 0,178 & 0,179 & 0,040 \\
\hline 4 & 35 & 0,130 & 0,315 & 0,151 & 0,161 & 0,181 & 0,174 & 0,141 \\
\hline 5 & 35 & 0,137 & 0,280 & 0,151 & 0,159 & 0,174 & 0,166 & 0,022 \\
\hline 6 & 9 & 0,046 & 0,259 & 0,095 & 0,154 & 0,175 & 0,143 & 0,070 \\
\hline 7 & 6 & 0,046 & 0,182 & 0,148 & 0,159 & 0,167 & 0,143 & 0,048 \\
\hline
\end{tabular}

n: número de pacientes Min: dato mínimo en el grupo

Max: dato máximo en el grupo $\mathrm{X}$ : promedio
Q1: percentil 25

Q2: percentil 50

Q3: percentil 75

DE: desviación estándar
Kruskal-Wallis entre todos los grupos: $p=0,0001$ Kruskal-Wallis, exceptuando el grupo 1: 0,8203 
Se pueden definir dos poblaciones según sus niveles de IgG específica contra E. histolytica, pacientes con AHA y los otros grupos (gráfico 1) con una distribución bimodal.

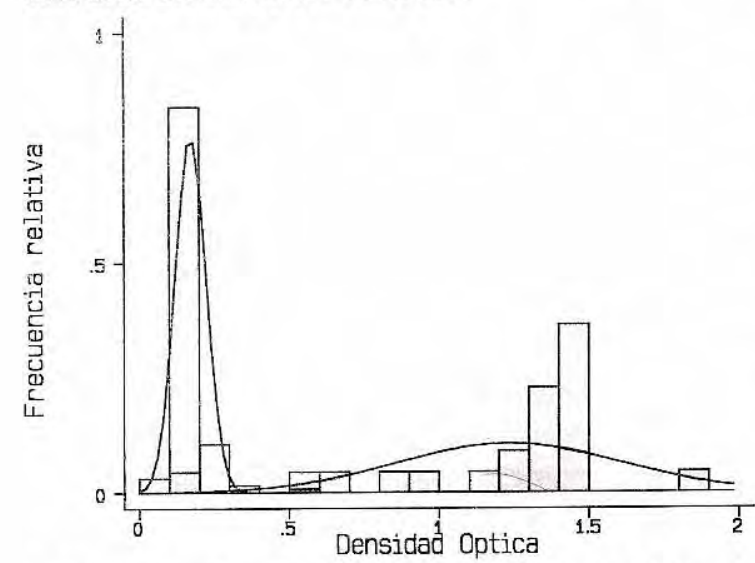

Gráfico 1. Distribución de los valores de densidad óptica de los sueros de pacientes con absceso hepático amebiano (grupo 1) y los otros grupos (grupos 2 al 7) en la prueba de Elisa para detección de IgG específica contra E. hystolytica.

\section{Capacidad discriminatoria de la prueba}

En el gráfico 2 se muestra la curva ROC para los valores agrupados; el área para esta forma de análisis es de 0,9791. El área bajo la curva para los datos sin agrupar es igual a 0,9941, dato muy cercano al ideal que es 1.

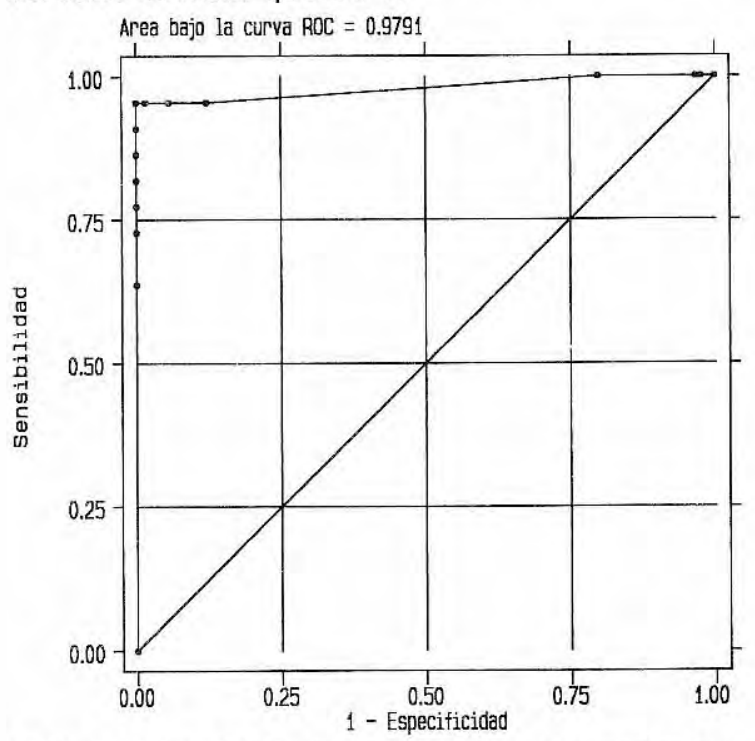

Gráfico 2. Curva ROC y área bajo la curva para las DO de la prueba de Elisa, agrupadas por 0,05 de DO para la detección de anticuerpos IgG contra E. histolytica en el diagnóstico de absceso hepático amebiano.

\section{Discusión}

Debido a la baja sensibilidad de la biopsia o aspiración hepática como prueba de referencia para el diagnóstico de absceso hepático amebiano, una buena alternativa es la detección de anticuerpos séricos IgG contra la ameba por su facilidad de detección por las pruebas serológicas actuales comparado con la detección de otras clases de inmunoglobulinas como $\lg M$ o $\lg E$ (4) y por su cinética de producción en el transcurso de la enfermedad. Estos anticuerpos empiezan a producirse intensamente a partir de la primera semana de iniciación de los síntomas y sus niveles se incrementan hasta cuatro semanas después del tratamiento. Posteriormente, pueden permanecer estables por un largo período de tiempo (9 a 15 meses) o declinar lentamente. Un incremento en la concentración de IgG después del tercer mes constituye un signo de mal pronóstico, indicativo de persistencia o recurrencia del absceso (11).

Sin embargo, cualquier prueba serológica requiere de una cuidadosa estandarización y evaluación de resultados para garantizar su real aplicabilidad en el diagnóstico de la enfermedad y una interpretación adecuada de sus resultados.

En la estandarización de esta prueba de ELISA para la detección de anticuerpos séricos lgG específicos contra $E$. histolytica, utilizamos una fase sólida con alta capacidad de adherencia con el objetivo de aumentar el nivel de captación del antígeno y así aumentar la detección de anticuerpos. Sin embargo, al usar esta clase de platos de microtitulación se presentaron varios inconvenientes principalmente en el paso de bloqueo de los sitios libres de antígeno (este bloqueo se realiza para disminuir las uniones inespecíficas del suero y conjugado a la fase sólida). Utilizando albúmina bovina (Sigma Chemical Co.) o leche descremada (skim milk, Difco Laboratories) a una concentración de 2 a $6 \%$ se presentaban altas lecturas de densidad óptica de fondo (DO de los blancos). Finalmente, usando albúmina de huevo se pudieron obviar estos inconvenientes; las lecturas de los blancos fueron cercanas a 0 y se detectaron $21 / 22$ 
pacientes con sospecha de AHA. El único paciente que presentó lecturas cercanas a $0(0,199$ DO) en esta prueba de ELISA-IgG era un paciente de 57 años de edad, de sexo masculino, quien presentaba un absceso hepático único de 7 centímetros de diámetro mayor en el lóbulo derecho que respondió al tratamiento con metronidazol y no presentaba factores de riesgo para AHP (16). Entonces, se recomienda la utilización de esta fase sólida teniendo en cuenta la importancia de la fase de bloqueo con albúmina de huevo.

Para la selección de las diluciones óptimas de suero y conjugado enzimático, además del nivel de discriminación positivo-negativo y de la DO de fondo en cada una de las diluciones, se tuvo en cuenta que la DO del pool de sueros positivo fuera aproximadamente la mitad del rango de DO detectado por el equipo $(0$ a $3,1,5)$, una característica importante para evaluar en futuros trabajos si existe alguna correlación entre los niveles de IgG específica y la severidad de la enfermedad puesto que por esta técnica se puede detectar un amplio rango de niveles de esta inmunoglobulina (17).

Además, el procedimiento fue estandarizado para realizarlo completamente en aproximadamente 7 horas (un Elisa convencional dura aproximadamente 26 horas, incluyendo la sensibilización de la fase sólida con el antígeno) lo cual podría permitir su aplicación en urgencias hospitalarias.

La evaluación de la prueba se encuentra en fase III-IV según la clasificación de Nierenberg y Feinstein (13) en la cual la prueba diagnóstico es evaluada en un amplio espectro de casos de la enfermedad (el grupo 1 incluía pacientes con AHA de distintos grados de severidad) y personas completamente sanas (grupos 2, 3, 4 y 5) o aquellas que han tenido contacto con $E$. histolytica (grupos 3, 4 y 7). Se determinó que las infecciones intestinales por esta ameba (aunque en el grupo 3 puede haber individuos infectados con $E$. dispar) no presentan niveles detectables de IgG específica contra E. histolytica por esta prueba. Sin embargo, es importante ampliar la cantidad de personas estudiadas en estos grupos.
Así mismo, se debe ampliar la cantidad y el espectro de pacientes en el grupo 6 puesto que este grupo incluye las entidades clínicas con las que realmente se debe hacer el diagnóstico diferencial de AHA (AHP, principalmente) para poder realizar la fase $V$ de la evaluación (13). En esta fase se deben evaluar otras enfermedades diferentes a AHA (entidades clínicas cuyas características clínicas sean semejantes a la enfermedad estudiada, por ejemplo, AHP, carcinoma hepático, hepatitis viral, cirrosis) y otras entidades que puedan ser interferentes potenciales de la prueba. En ninguno de los artículos revisados donde se evalua una prueba para el diagnóstico de $\mathrm{AHA}$, se incluye un grupo significativo de estos pacientes $(3,4,7-9,18)$ exceptuando el estudio revisado por Shenai y col. (10) en el cual se incluyen 6 pacientes con absceso hepático piógeno, 6 pacientes con hepatitis y 11 pacientes con hepatocarcinoma. Con este tipo de pacientes, se podrían obtener valores reales de sensibilidad, especificidad y valores predictivos y nos darían una estimación real de la capacidad discriminatoria de la prueba para obtener el verdadero valor del área bajo la curva y no el casi ideal que informamos aquí.

Este trabajo representa un estudio preliminar en la evaluación de una prueba diagnóstica para absceso hepático amebiano que debe continuarse, ampliando el espectro y número de pacientes en los diferentes grupos. Una vez evaluada la prueba, se podría realizar un estudio buscando la correlación entre los niveles de IgG específica y la severidad del absceso en la búsqueda de indicadores de pronóstico de la enfermedad y su seguimiento.

\section{Agradecimientos}

El presente trabajo fue realizado con aportes de la Dirección General de Investigaciones, la Escuela de Bacteriología y el Laboratorio de Inmunología de la Facultad de Salud de la Universidad Industrial de Santander. Agradecemos al doctor R.S. Nicholls del Laboratorio de Parasitología del Instituto Nacional de Salud y al personal del Hospital Ramón González Valencia de la ciudad de Bucaramanga por proporcionarnos las historias clínicas y los sueros para los grupos 1 y 6 . 


\section{Referencias}

1. Ravdin Jl. Amebiasis. Clin Inf Dis 1995;20:1453-6.

2. Botero D, Restrepo M. Amibiasis. En: Botero D, Restrepo M, editores. Parasitosis humanas. Medellín: Colombia: Corporación para Investigaciones Biológicas; 1995:43 y 48.

3. Restrepo MI, Restrepo Z, López C, Villareal E, Aguirre A, Restrepo M. Diagnostic tests for amoebic liver abscess: comparison of enzyme-linked immunosorbent assay (ELISA) and counterimmunoelectrophoresis (CIE). Rev Soc Bras Med Trop 1996;29:27-2.

4. Shetty N, Nagpal S, Subba Rao PV, Schroder H. Detection of $\lg \mathrm{G}, \operatorname{lgA}$, IgM and IgE in antibodies in invasive amoebiasis in endemic areas. Arch Invest Méd 1990;21 (Supl 1):41-6.

5. Robles P, Castaño A, Montoya MN. Absceso hepático amebiano, diagnóstico serológico. Temas Microbiológicos 1993;14:1-5.

6. JacksonTFHG, Anderson CB, Simjee AE. Serological differentiation between past and present infection in hepatic amoebiasis. Trans Roy Soc Trop Med Hyg 1984; 78:342-5.

7. Aceti A, Pennica A, Caferro M, Celestino D, Paparo BS, Sebastiani A. Application of time resolved fluoroimmunoassay (TR-FIA) for the diagnosis of invasive amoebiasis. Trans Roy Soc Trop Med Hyg 1987;81:7647.

8. Lotter H, Mannweiler E, Tannich E. Crude or recombinant proteins applied to latex agglutination, complement fixation and enzyme-linked immunosorbent assays for the serodiagnosis of invasive amebiasis. Trop Med Parasitol 1993;44:277-80.

9. Abd-Alla MD, El-Hawey AM, Ravdin Jl. Use of an enzyme-linked immunosorbent assay to detect antiadherence protein antibodies in sera of patients with invasive amebiasis in Cairo, Egypt. Am J Trop Med Hyg
$1992 ; 47: 800-4$

10. Shenai BR, Komalan BI, Arvind AS, Krishnaswamy PR, Subba Rao PV. Recombinant antigen-based avidinbiotin microtiter enzyme-linked immunosorbent assay for serodiagnosis of invasive amebiasis. J Clin Microb 1996; 34:828-3.

11. Pinon JM, Poirriez J, Remy G, Lepan H. Immunological studies in amebiasis: isotypic characterization of specific antibodies by enzyme-linked immunofiltration assay. Am J Trop Med Hyg 1987;37:290-5.

12. Okasaki M, Okasazi M, Miranda P, Neto J, Diegues V, Alves $\mathrm{J}$, et al. Parasitological and serological studies on amoebiasis and other intestinal parasitic infections in Recife and its suburban area, Northeast Brazil. Rev Inst MedTrop Sao Paulo 1988;30:313-1.

13. Nierenberg AA, Feinstein AR. How to evaluate a diagnostic marker test, lessons from the rise and fall of dexamethasone suppression test. JAMA 1988;259:16992.

14. Beck JR, Shultz EK. The use of relative operating characteristic (ROC) curves in test performance evaluation. Arch Pathol Lab Med 1986;110:13-20.

15. Garduño J, Martínez MC, Rendón E, Fajardo A, Hernández DM, Muñoz $\mathrm{O}$. Rendimiento diagnóstico de las pruebas inmunológicas en absceso hepático amibiano a través de curvas de características operantes del receptor. Rev Inv Clin 1992;44:373.

16. Lee KT, Sheen PC, Chen JS, Ker CG. Pyogenic liver abscess: multivariate analysis of risk factor. World J Surg 1991;15:372-7.

17. Arteaga HJ, Burbano LF, Contreras JP, Leal CV, Trujillo CM. Correlación clínico-inmunológica en absceso hepático amebiano. Med UIS 1995;9:116-20.

18. Ximenez C, Leyva O, Moran P, Ramos F, Melendro EI, Ramiro M, et al. Entamoeba histolytica: antibody response to recent and past invasive events. Ann Trop Med Parasitol 1993;87:31-9. 